ISSN 0001-6012/2020/62/4/181-186 Acta Médica Costarricense, (C) 2020 Colegio de Médicos y Cirujanos de Costa Rica

\title{
Original
}

\section{Efecto de la rehabilitación pulmonar sobre la función respiratoria y la capacidad de ejercicio en personas con enfermedad pulmonar crónica}

\author{
(Effect of pulmonary rehabilitation on respiratory function and \\ exercise capacity in people with chronic lung disease)
}

Franklin Heyden-López ${ }^{1}$, Derby Muñoz-Rojas²

\section{Resumen}

$\begin{array}{lcr}\text { Afiliación } & \text { de } & \text { los } \\ \text { autores: } & \text { 1Programa } & \text { de }\end{array}$ reacondicionamiento físico para adultos mayores con enfermedad pulmonar crónica, Hospital Nacional de Geriatría y Gerontología- Caja Costarricense de Seguro Social, Costa Rica. ${ }^{2}$ Centro de investigación en cuidado de enfermería, Escuela de Enfermería, Universidad de Costa Rica.

Trabajo realizado en: Hospital Nacional de Geriatría y Gerontología- Caja Costarricense de Seguro Social, Costa Rica.

Abreviaturas: CVF, Capacidad vital forzada; C6M, caminata de seis minutos; $\mathrm{VO}_{2}$, consumo de oxígeno; $\mathrm{FEF}_{25-75^{\prime}}$ flujo espiratorio forzado 25-75; HNGG-CCSS, Hospital Nacional de Geriatría y Gerontología-Caja Costarricense de Seguro Social; PAM, personas adultas mayores; PEmáx, presión espiratoria máxima; PImáx, presión inspiratoria máxima; PRFAMEPC, programa de reacondicionamiento físico para adulto mayor con enfermedad pulmonar crónico; $P E$, prueba de ejercicio; $\mathrm{VEF}_{\mu} \mathrm{CVF}_{\text {, }}$ relación entre el volumen espiratorio forzado en un segundo y la capacidad vital forzada; RP, rehabilitación pulmonar; RVPECP, reserva ventilatoria en la prueba de ejercicio cardiopulmonar; UAPECP, umbral anaeróbico ventilatorio en la prueba de ejercicio cardiopulmonar; $\mathrm{VEF}_{1}$, volumen espiratorio forzado en un segundo; VEmáx, volumen minuto ventilatorio máximo; VVM, ventilación voluntaria máxima.

凶fheyden@ccss.sa.cr
Justificación y objetivo: los problemas pulmonares crónicos son una de las principales causas de discapacidad en las personas adultas mayores. Estas enfermedades afectan negativamente la calidad de vida y la capacidad funcional de las personas, debido a la interacción entre el proceso normal de envejecimiento y la fisiopatología. Sin embargo, los programas de rehabilitación pulmonar parecen ser una opción terapéutica efectiva para tratar esta problemática. A pesar de esto, en Costa Rica no es una posibilidad disponible en todos los centros de salud y existe limitada evidencia sobre su efectividad. Este estudio intenta identificar el efecto de un programa de rehabilitación pulmonar sobre la función respiratoria y la capacidad de ejercicio de personas adultas mayores con enfermedad pulmonar crónica.

Métodos: se realizó un análisis retrospectivo del efecto de un programa de ejercicio físico sobre la función respiratoria y la capacidad de ejercicio en 53 personas adultas con enfermedad pulmonar crónica. Los datos fueron recolectados en el Hospital Nacional de Geriatría y Gerontología-Caja Costarricense de Seguro Social, de personas que participaron en el programa entre enero 2012 y diciembre 2017.

Resultados: hubo mejoras estadísticamente significativas en la función respiratoria $(p<0,001)$ y la capacidad de ejercicio $(\mathrm{p}<0,001)$ de los sujetos, luego de participar en el programa.

Conclusión: el programa de rehabilitación pulmonar mejoró la función respiratoria y la capacidad de ejercicio.

Descriptores: adulto mayor, capacidad cardiovascular, ejercicio físico, enfermedad pulmonar.

\section{Abstract}

Background and aim: Chronic lung problems are one of the main causes of disability in older adults. These diseases negatively affect the quality of life and functional capacity of these people, due to the interaction between the normal aging process and the pathophysiology. However, pulmonary reconditioning programs seem to be an effective therapeutic alternative to treat this problem. Despite this, in Costa Rica it is not an option available in all health centers and there is limited evidence on its effectiveness. Therefore, this study attempts to identify the effect of pulmonary rehabilitation on the respiratory function and aerobic capacity of exercise among older adults with chronic obstructive pulmonary disease.

Methods: For which a retrospective analysis of the effect of a physical exercise program on ventilatory function and aerobic capacity of exercise in 53 adults with chronic lung disease wasperformed. Data 
were collected at the National Hospital of Geriatrics and Gerontology - Caja Costarricense de Seguro Social, of people who participated in the program between January 2012 and December 2017.

Results: It was determined that there were statistically significant improvements in respiratory function $(p<0.001)$ and capacity of exercise $(p<0.001)$ of the subjects after participating in the program.

Conclusion: The pulmonary rehabilitation program improved respiratory function and exercise capacity.

Keywords: cardiorespiratory fitness, exercise, lung disease, older adult.

Fecha recibido: 14 de enero 2020

Fecha aprobado: 27 de agosto 2020

Las enfermedades respiratorias crónicas son un serio problema de salud pública en Costa Rica. Datos del Ministerio de Salud (2014), estiman que al año ocurren cerca de 880 muertes por enfermedades respiratorias crónicas, en donde las personas adultas mayores (PAM) son desproporcionadamente afectadas, ya que la cantidad de fallecimientos se incrementa de manera seria a partir de los 50 años. Las estadísticas del Hospital Nacional de Geriatría y Gerontología, de la Caja Costarricense de Seguro Social (HNGG-CCSS, 2016), indican que las enfermedades respiratorias ocupan el sexto lugar entre las causas de egreso en ese centro hospitalario. Este tipo de problemas fue identificado además como la causa de defunción del 18,5\% de las muertes reportadas en ese hospital, situación que asemeja los patrones internacionales, posicionando mundialmente estas enfermedades como una de las principales causas de discapacidad severa en las PAM. ${ }^{1}$

Las manifestaciones y las complicaciones de las enfermedades pulmonares crónicas en las PAM, son el resultado de la interacción entre el proceso normal de envejecimiento y la fisiopatología característica de este tipo de padecimientos, lo que produce que las PAM que sufren de este tipo de problemas, tengan una serie de complicaciones que van desde cuadros de disnea, acúmulo de secreciones y cambios en la morfología cardiopulmonar, hasta serios problemas de disminución en la capacidad de ejercicio y la masa muscular, lo que finalmente impacta de forma negativa la calidad de vida de los individuos. ${ }^{2}$ Por ejemplo, con respecto a este deterioro funcional, se ha documentado en la bibliografía la espiral de la disnea (AACVPR, 2011), en la cual las PAM con problema pulmonar presentan disnea, que los lleva a limitar sus actividades físicas, lo que a su vez deteriora su función muscular, causando debilidad muscular, que a su vez incrementa la sensación de disnea. ${ }^{3}$

Con el fin de disminuir estos procesos de deterioro funcional, se ha recomendado internacionalmente la implementación de programas de rehabilitación pulmonar (RP), ${ }^{3-5}$ entendiéndose esta como una intervención basada en la evidencia, multidisciplinaria, para personas con enfermedades pulmonares crónicas, que pretende reducir los síntomas, optimizar su funcionalidad, aumentar su participación en actividades de la vida diaria y disminuir la utilización de recursos sanitarios. ${ }^{6}$

Estos programas están conformados por diferentes componentes, incluyendo una evaluación inicial y final con prueba de función respiratoria, entrenamiento muscular con ejercicio aeróbico y de fuerza de brazos y piernas, entrenamiento de los músculos respiratorios, educación, fisioterapia respiratoria, terapia ocupacional, soporte psicosocial e intervención nutricional. ${ }^{3,5-7}$

Incluso, se ha documentado que los programas de RP enfocados en el ejercicio físico poseen múltiples beneficios para la PAM con problemas pulmonares crónicos. Diferentes estudios han encontrado que disminuyen la percepción de disnea (grado de recomendación $1^{\mathrm{a}}$ ), mejoran la calidad de vida relacionada con la salud (grado de recomendación 1A), reducen el número de días de hospitalización (grado de recomendación: 2B), ${ }^{3}$ así como también optimizan la tolerancia al ejercicio. ${ }^{5}$

En Costa Rica, recientemente se ha empezado a incursionar en la RP. En el caso del HNGG-CCSS, se cuenta un Programa de Reacondicionamiento Físico para Adulto Mayor con Enfermedad Pulmonar Crónica, ubicado en la fase dos de un Programa de RP. El programa está organizado en módulos: el primero corresponde a la evaluación de la persona pre y postparticipación en el programa; el segundo se enfoca en el trabajo específico organizado en 12 semanas (24 sesiones) de trabajo dirigido y sin interrupción, que incluye ejercicio aeróbico de 12 semanas, trabajo contra resistencia (pesas) de 10 semanas, trabajo funcional de 10 semanas y fisioterapia respiratoria de 12 semanas (i.e., reeducación respiratoria, terapia expansión pulmonar con uso de inspirometría incentiva y dispositivos de presión espiratoria positiva, así como entrenador de músculo inspiratorio de umbral).

A pesar de que se ha documentado los beneficios de este tipo de intervenciones, en el país no es una opción terapéutica que se ofrece en todos los centros de salud, y existe aún menos disponibilidad de programas de rehabilitación pulmonar para PAM con enfermedades pulmonares crónicas. Por tal motivo, esta investigación pretende mejorar el conocimiento sobre la efectividad de este tipo de intervenciones, al determinar el efecto de un programa de rehabilitación pulmonar sobre la función respiratoria y la capacidad de ejercicio.

\section{Métodos}

Esta investigación utilizó un diseño descriptivo comparativo retrospectivo, en el cual se realizó un análisis de registros 


\section{Rehabilitación pulmonar en enfermedad pulmonar crónica / Heyden-López, Muñoz-Rojas}

médicos de las personas participantes en el Programa de Reacondicionamiento Físico para Adulto Mayor con Problema Pulmonar Crónico (PRFAMEPC) del HNGG-CCSS, en donde se atendió a 57 usuarios entre 2012 y 2017. Se trabajó con los datos de 53 personas, cuyos registros se encontraban en el Laboratorio de Función Pulmonar del Servicio de Terapia Respiratoria. Estas personas fueron: 1. identificadas como mayores de 65 años, 2. participantes en el programa entre enero 2012 y diciembre 2017, 3. identificadas como obstructivas (i.e., valores espirométricos con $\mathrm{VEF}_{1} / \mathrm{CVF}$ menor del 70 \% del predicho y CVF menor del $80 \%$ del predicho) o restrictivos (valores espirométricos con $\mathrm{VEF}_{1} / \mathrm{CVF}$ mayor o igual al $70 \%$ del predicho y CVF menor del $80 \%$ del predicho). No hubo restricción asociada al género o la etnia. De los registros se tomaron los datos sobre la función respiratoria y la capacidad de ejercicio, así como sobre el sexo y la edad. Todos los procedimientos de esta investigación fueron aprobados por el Comité Ético Científico del HNGG-CCSS, protocolo HNGGCEC 04-2018, y fueron inscritos en el Consejo Nacional de Investigación en Salud.

Los datos extraídos correspondieron a la información recolectada durante la evaluación inicial y final del programa. Para efectos de este análisis se recolectaron los siguientes datos:

La función respiratoria se valoró mediante las siguientes pruebas de función pulmonar: capacidad vital forzada (CVF), expresada en litros y como porcentaje del predicho, valor normal $\geq 80 \%$ del predicho;,9 volumen espirado forzado en un segundo (VEF1), expresado en litros y como porcentaje del predicho, valor normal $\geq 80 \%$ del predicho; ${ }^{8,9}$ relación entre el volumen espiratorio forzado en un segundo y la capacidad vital forzada (VEF1/CVF), valor normal > $70 \%$ del predicho; 8,9 flujo espiratorio forzado 25 - $75\left(\mathrm{FEF}_{25-75}\right)$, medido en litros y en porcentaje del predicho, valor normal > $65 \%$ del predicho; ${ }^{8,9}$ presión inspiratoria máxima (PImáx), expresada en kilopascales y como porcentaje del predicho, valores normales $\geq 80 \%$ del predicho; presión espiratoria máxima (PEmáx), expresada en kilopascales y como porcentaje del predicho, valor normal $\geq 80$ \% del predicho; ${ }^{9}$ ventilación voluntaria máxima (VVM) expresada en litros/min y como porcentaje del predicho, valor normal $\geq 80 \%$ del predicho. ${ }^{9}$

La capacidad de ejercicio se valoró mediante las siguientes pruebas: caminata de seis minutos, shuttle test y prueba de ejercicio cardiopulmonar. La caminata de seis minutos (C6M) es una prueba de campo submáxima que consiste en medir la distancia recorrida en seis minutos, expresada en metros recorridos y porcentaje del predicho, valor normal > $80 \%$ del predicho.9 Shuttle test es una prueba de campo máxima, llamada también prueba de lanzadera. Se trata de una prueba de caminata progresiva, que consiste en caminar un tramo de 10 metros, a una velocidad que aumenta cada minuto. La prueba finaliza al lograr la máxima velocidad posible por el paciente. ${ }^{9}$ La prueba de ejercicio cardiopulmonar es máxima, incremental, que utiliza un analizador de gases espirados, y

\begin{tabular}{|c|c|c|c|c|c|c|c|c|c|c|c|c|c|}
\hline \multicolumn{14}{|c|}{ Semanas } \\
\hline 1 & 2 & 3 & 4 & 5 & 6 & 7 & 8 & 9 & 10 & 11 & 12 & 13 & 14 \\
\hline \multirow[t]{5}{*}{ Evaluación inicial } & & & & & & & & & & & & & Evaluación final \\
\hline & \multicolumn{12}{|c|}{$\begin{array}{l}\text { Trabajo aeróbico ( } 12 \text { semanas) } \\
\text { Tipos de ejercicio: caminadora, cicloergómetro, gradas. Frecuencia: } 2 \text { v/s } \\
\text { supervisado. Intensidad: entre el } 50 \text { y el } 80 \% \text { del VO2R. Tempo: } 15 \text { a } 30 \\
\text { minutos por sesión. }\end{array}$} & \\
\hline & & & \multicolumn{10}{|c|}{$\begin{array}{l}\text { Trabajo contra resistencia (pesas) (10 semanas) } \\
\text { Tipos de ejercicio: mancuernas, extensión-flexión de rodilla } \\
\text { y press de pecho sedente (en biomecánicos de movimientos } \\
\text { dirigidos). Frecuencia: } 2 \text { v/s supervisado. Intensidad: } 50 \text { al } 70 \% \\
\text { de 8-12 RM. Tempo: } 10 \text { a } 15 \text { minutos por sesión. }\end{array}$} & \\
\hline & & & \multicolumn{10}{|c|}{$\begin{array}{l}\text { Trabajo funcional (10 semanas) } \\
\text { Tipos de ejercicio: movimientos corporales que incluyó cambios } \\
\text { de nivel, tracción, empuje y rotación con pesos libres o propio } \\
\text { peso. Frecuencia: } 1 \text { v/s supervisado. Intensidad: } 3-4 / 10 \text { escala } \\
\text { Borg modificada. Tempo: } 10 \text { a } 15 \text { minutos por sesión }\end{array}$} & \\
\hline & \multicolumn{12}{|c|}{$\begin{array}{l}\text { Fisioterapia respiratoria (12 semanas) } \\
\text { Tipo: reeducación ventilatoria (respiración diafragmática, labios fruncidos, } \\
\text { ejercicios enfocados en los músculos de la fase inspiratoria), expansión } \\
\text { pulmonar (inspirómetro incentivo y dispositivo de presión espiratoria positiva } \\
\text { no oscilante (DPEP)), entrenador de músculo inspiratorio de umbral (EMI). } \\
\text { Frecuencia: } 2 \text { v/d, } 3 \text { v/s en el hogar. Intensidad: DPEP (6-12 cmH2O), EMI } \\
\text { (40 - } 60 \% \text { de la PImáx) series: 4-6, repeticiones: } 10-20 \text {. Duración } 15 \text { a } 20 \\
\text { minutos por sesión. }\end{array}$} & \\
\hline \multicolumn{14}{|c|}{$\begin{array}{l}\text { Figura 1. Distribución del Programa de Reacondicionamiento Físico para Adulto Mayor con Enfermedad Pulmonar Crónica del HNGG-CCSS } \\
\text { Nota: PImáx presión inspiratoria máxima; RM repeticiones máximas v/d veces por día; v/s veces por semana; VO2R consumo de oxígeno reserva } \\
\text { Elaboración propia con base en los datos del PRFAMEPC }\end{array}$} \\
\hline
\end{tabular}


consiste en los registros de consumo de oxígeno $\left(\mathrm{VO}_{2}\right)$ medido en $\mathrm{ml} / \mathrm{kg} / \mathrm{min}$ y como porcentaje del predicho; umbral anaeróbico ventilatorio medido como porcentaje del $\mathrm{VO}_{2}$ máximo; volumen minuto ventilatorio máximo medido en L/ min y reserva ventilatoria. ${ }^{9}$

E1 PRFAMEPC (Figura 1) se ejecutó durante dieciséis semanas, distribuidas de la siguiente manera: dos semanas de evaluación, una inicial y otra final, en la que se mide la antropometría, disnea, función respiratoria, capacidad de ejercicio, fuerza y resistencia muscular de miembros inferiores y superiores, calidad de vida relacionada con la salud y evaluación multidimensional. Y catorce semanas de trabajo, que incluyeron trabajo aeróbico, pesas, trabajo funcional y fisioterapia respiratoria. Todos los participantes realizaron los mismos ejercicios.

\section{Análisis estadístico}

Los datos fueron extraídos manualmente a un formulario diseñado para el estudio, y luego exportados a una base de datos en SPSS 24.0®. Se generaron estadísticas descriptivas para la muestra. Además, se ejecutaron análisis univariados para identificar el efecto del programa, incluyendo t-student. Los datos perdidos fueron identificados en el sistema $e$ imputados.

\section{Resultados}

La edad promedio de los 53 adultos mayores fue de 77,60 \pm 5,78 años; 33 hombres (62 \%), 20 mujeres (38 \%). 45 (85 $\%)$ son personas con problema obstructivo y 8 (15\%), con problema pulmonar restrictivo.

Tal y como se desprende del Cuadro 1, la función respiratoria de los participantes mejoró en algunos de los indicadores, al comparar las evaluaciones finales con respecto a las iniciales. En particular, de forma estadísticamente significativa, se detectó una mejora en la $\mathrm{FEF}_{25.75}$ (obstrucción vía aérea pequeña, diferencia promedio $=0,16, p=0,033$ ), la PImáx (fuerza muscular inspiratoria, diferencia promedio $=0,65, p=0,001$ ), la PEmáx (fuerza muscular espiratoria, diferencia promedio predicho $=4,92, p=0,010$ ), la VVM (resistencia muscular, diferencia promedio $=3,28, p=0,050$ ) y una disminución en la relación $\mathrm{VEF}_{1} / \mathrm{CVF}$ (diferencia promedio $=3,05, p=0,027$ ).

Se comprobó que el programa tuvo un efecto positivo en la capacidad de ejercicio, según los resultados de las evaluaciones en las pruebas de caminata y el Shuttle test. Como se desprende del Cuadro 2, los participantes aumentaron significativamente su desempeño en la C6M (diferencia promedio $=43,16$, $\mathrm{p}<0,001$ ), el Shuttle test (diferencia promedio $=23,5, \mathrm{p}=0,016$ ),

\begin{tabular}{|c|c|c|c|c|c|c|c|}
\hline & $n$ & Pre $\pm D E$ & Post $\pm D E$ & $\begin{array}{c}\text { Dif. } \\
\text { promedio }\end{array}$ & $t$ & $g l$ & $p$ \\
\hline CVF (L) & 53 & $2,30 \pm 0,92$ & $2,40 \pm 1,03$ & $-0,084$ & $-1,64$ & 52 & 0,108 \\
\hline CVF (\% predicho) & 53 & $82,87 \pm 27,81$ & $85,55 \pm 30,88$ & $-2,68$ & $-1,34$ & 52 & 0,186 \\
\hline VEF1 (L) & 53 & $1,29 \pm 0,52$ & $1,25 \pm 0,56$ & 0,034 & 1,06 & 52 & 0,293 \\
\hline VEF1 (\% predicho) & 53 & $71,34 \pm 25,21$ & $71,85 \pm 26,32$ & $-0,509$ & $-0,297$ & 52 & 0,768 \\
\hline Relación VEF ${ }_{1} / C V F$ & 53 & $58,87 \pm 18,63$ & $55,83 \pm 20,01$ & 3,05 & 2,28 & 52 & 0,027 * \\
\hline $\mathrm{FEF}_{25-75}(\mathrm{~L})$ & 53 & $0,76 \pm 0,60$ & $0,92 \pm 0,99$ & $-0,16$ & $-2,19$ & 52 & $0,033^{*}$ \\
\hline $\begin{array}{l}\mathrm{FEF}_{25-75} \\
\text { (\% predicho) }\end{array}$ & 53 & $40,32 \pm 31,14$ & $44,91 \pm 50,68$ & $-4,58$ & $-1,32$ & 52 & 0,191 \\
\hline PImáx (Kpa) & 52 & $4,99 \pm 2,60$ & $5,64 \pm 2,46$ & $-0,646$ & $-3,66$ & 51 & 0,001 * \\
\hline PImáx (\% predicho) & 52 & $58,35 \pm 27,13$ & $65,83 \pm 24,88$ & $-7,48$ & $-3,39$ & 51 & 0,001 * \\
\hline PEmáx (Kpa) & 51 & $6,79 \pm 3,10$ & $7,13 \pm 2,81$ & $-0,333$ & $-1,08$ & 50 & 0,286 \\
\hline $\begin{array}{c}\text { PEmáx } \\
\text { (\% predicho) }\end{array}$ & 51 & $42,61 \pm 17,39$ & $47,53 \pm 18,29$ & $-4,92$ & $-2,68$ & 50 & 0,010 * \\
\hline VVM (L/min) & 51 & $48,51 \pm 21,45$ & $51,78 \pm 24,24$ & $-3,28$ & $-2,01$ & 50 & 0,050 * \\
\hline VVM (FR) & 51 & $74,80 \pm 24,71$ & $72,96 \pm 22,63$ & 1,84 & 0,599 & 50 & 0,552 \\
\hline $\begin{array}{c}\text { VVM } \\
\text { (\% predicho) }\end{array}$ & 51 & $54,04 \pm 20,95$ & $57,06 \pm 20,77$ & $-3,02$ & $-1,64$ & 50 & 0,108 \\
\hline
\end{tabular}


Rehabilitación pulmonar en enfermedad pulmonar crónica / Heyden-López, Muñoz-Rojas

de la misma forma que en el umbral anaeróbico ventilatorio de la prueba de ejercicio cardiopulmonar (diferencia promedio= $9,77, p=0,035)$.

\section{Discusión}

Los resultados mostraron que los participantes mejoraron su función respiratoria luego de participar en el Programa de Reacondicionamiento Físico para Adulto Mayor con Enfermedad Pulmonar Crónica. Los cambios en la PImáx y la VVM siguieren que el PRFAMEPC provocó una mejora en esta variable al incrementar la fuerza de la musculatura inspiratoria y resistencia muscular ventilatoria. Esto concuerda con lo documentado en investigaciones similares, en las cuales encontraron que el entrenamiento de la musculatura inspiratoria impactaba positivamente la fuerza muscular, la resistencia a la fatiga de los músculos repiratorios, la capacidad de ejercicio y la calidad de vida, así como una disminución en la disnea.,10-15

En este estudio se encontró además que los cambios en la presión inspiratoria fueron de 0,647 $\mathrm{Kpa}(6,6 \mathrm{cmH} 2 \mathrm{O})$ inferiores a los $13 \mathrm{cmH} 2 \mathrm{O}$ de diferencia mínima reportados por Basso-Vanelli (2016). Aun así, en este estudio hay ganancia en la fuerza, que se puede deber al entrenamiento de los músculos respiratorios y los principios de especificidad y sobrecarga empleados en el programa, los que contribuyen a que se presenten cambios estructurales, funcionales y sistémicos, llevando a mejorar la capacidad de ejercicio y reducir la disnea. ${ }^{5}$ Adicionalmente, el entrenamiento de los músculos inspiratorios (EMI) debe considerarse como un tratamiento adyuvante dentro del PRFAMEPC, sobre todo en pacientes con debilidad muscular con valores de PImáx menores de $60 \mathrm{cmH} 2 \mathrm{O}$, lo cual fue evidente en los valores obtenidos en la evaluación inicial. ${ }^{5}$

El aumento de la fuerza inspiratoria mejora el volumen de gas inspirado, que aun cuando no sea significativo, es importante durante la realización de actividades de la vida diaria. El componente de ejercicio físico del PRFAMEPC aumenta el volumen inspiratorio y reduce la hiperinflación dinámica. ${ }^{16} \mathrm{La}$ mejora de la fuerza espiratoria pudo haber mejorado el vaciado pulmonar, reduciendo así la hiperinsuflación dinámica. El trabajo instrumental con sistemas de PEP pudo haber mejorado la ventilación colateral, reducido el cierre temprano de la vía aérea y la hiperinsuflación dinámica. ${ }^{17}$

Los resultados mostraron una disminución en la relación $\mathrm{VEF}_{1} / \mathrm{CVF}$, que concuerda con la reducción no significativa del $\mathrm{VEF}_{1}$. Lo anterior se puede deber a varios componentes: el trabajo incluye personas con problemas obstructivos y restrictivos; los participantes con problemas restrictivos presentan un problema de fuelle que podría afectar el $\mathrm{VEF}_{\text {1. }}$ los pacientes lograrán una mejor medición de CVF en la espirometría, o a la progresión en la obstrucción de la vía aérea. Sin embargo, es importante destacar el hecho del aumento, aunque no significativo en la CVF y el $\mathrm{FEF}_{25.75}$, indicadores sugestivos de mejora en la capacidad pulmonar y condición de la vía aérea.

Similarmente, se comprobó que la capacidad de ejercicio mejoró luego de participar en el PRFAMEPC. La bibliografía menciona que la rehabilitación pulmonar mejora la capacidad funcional, la capacidad de ejercicio y la tolerancia al ejercicio de miembros inferiores y superiores. ${ }^{5,15}$ En este trabajo se evidenció el aumento en la distancia recorrida en la C6M y la prueba shuttle, con cambios de 43,16 y 23,5 metros, respectivamente. Se ha encontrado en estudios similares, que este tipo de programas puede mejorar la distancia recorrida en la C6M y reporta cambios de hasta 43,93 metros, ${ }^{16,18}$ con 35 metros como cambio mínimo clínicamente significativo, e incluso 26 metros en EPOC con una obstrucción grave ${ }^{7}$ y 49 y 54 metros como cambios mínimos clínicamente significativos. ${ }^{5}$ De la misma forma, para la prueba shuttle se han registardo cambios de hasta 47,5 metros. $^{7}$

Se observó también una mejora de $1,12 \mathrm{ml} / \mathrm{Kg} / \mathrm{min}$ en el $\mathrm{VO}_{2}$ y una variación significativa del umbral anaeróbico ventilatorio, lo cual puede ser el resultado de la capilarización de fibras musculares que ocurre por el ejercicio, mejorando la capacidad oxidativa y reduciendo la cantidad de lactatos y

\begin{tabular}{|c|c|c|c|c|c|c|c|}
\hline & $n$ & Pre \pm DE & Post $\pm \mathrm{DE}$ & Dif promedio & $\mathrm{t}$ & gl & $\mathrm{P}$ \\
\hline C6M (m) & 25 & $220,84 \pm 90,20$ & $264,00 \pm 102,88$ & $-43,16$ & $-4,98$ & 24 & $0,000 * *$ \\
\hline C6M (\% predicho) & 22 & $56,01 \pm 24,23$ & $68,82 \pm 28,41$ & $-12,73$ & $-5,12$ & 21 & $0,000 * *$ \\
\hline Shuttle (m) & 32 & $175,31 \pm 87,80$ & $198,81 \pm 98,95$ & $-23,50$ & $-2,54$ & 31 & $0,016^{*}$ \\
\hline VO2PECP (ml/Kg/x') & 12 & $9,82 \pm 3,19$ & $10,95 \pm 5,08$ & $-1,12$ & $-1,41$ & 11 & 0,187 \\
\hline VOZPECP (\% predicho) & 12 & $48,58 \pm 12,57$ & $53,67 \pm 20,83$ & $-5,08$ & $-1,33$ & 11 & 0,211 \\
\hline UAPECP (\% del VO2 máx) & 13 & $13,15 \pm 18,76$ & $22,92 \pm 22,28$ & $-9,77$ & $-2,38$ & 12 & $0,035^{*}$ \\
\hline VEmáxPECP (L) & 17 & $39,67 \pm 19,71$ & $39,18 \pm 18,74$ & 0,49 & 0,22 & 16 & 0,827 \\
\hline RVPECP & 17 & $19,17 \pm 28,80$ & $25,12 \pm 22,27$ & $-5,95$ & $-1,34$ & 16 & 0,197 \\
\hline
\end{tabular}


fatiga, lo que permite a la persona mejor tolerancia ante una carga de ejercicio. La bibliografía reporta variaciones en el consumo de oxígeno pico de $+2 \mathrm{ml} / \mathrm{Kg} / \mathrm{x}$ ' postintervención (evidencia grado B), ${ }^{19}$ y cambio de 100 - 105 segundos. ${ }^{7}$

Por su parte, las recomendaciones internacionales mencionan que la adición de un componente de entrenamiento de fuerza en un programa de RP, aumenta la masa y fuerza muscular con una evidencia $1 \mathrm{~A} .{ }^{3}$ El trabajo de los miembros superiores incrementa la resistencia de los brazos, modula la hiperinflación dinámica y reduce los síntomas de disnea y fatiga muscular, así como el ejercicio aeróbico de miembros inferiores incrementa la tolerancia al ejercicio. ${ }^{5}$

Con base en los resultados obtenidos se puede concluir que un programa de rehabilitación pulmonar para PAM con problemas pulmonares crónicos, debe combinar eficazmente la prescripción de ejercicios aeróbicos, de contrarresistencia, así como también trabajo funcional y fisioterapia respiratoria. Igualmente, el efecto positivo del programa en la función respiratoria y en la capacidad de ejercicio, parece ser el resultado de un manejo adecuado de cargas de trabajo y especificidad, lo que permitió una readaptación al esfuerzo físico y, por ende, una mejora en la tolerancia a este. Se debería realizar investigaciones futuras para examinar la efectividad de este programa con el fin de identificar con más detalle su efecto sobre otras variables de interés, como la función hemodinámica, además de que existen particularidades fisiológicas asociadas a los problemas restrictivos y obstructivos que deben ser revisadas durante la evaluación y prescripción.

Estos resultados deben ser interpretados con precaución, ya que el estudio tuvo algunas limitaciones que deben ser reportadas, incluyendo que los datos fueron recolectados primariamente para fines prácticos y no investigativos, se contó con una muestra limitada de participantes, por lo que se realizó un análisis conjunto de participantes con problemas obstructivos y restrictivos, y no fue posible separarlos, debido a la poca cantidad de participantes restrictivos.

Al respecto de las implicaciones de este trabajo, los hallazgos podrían utilizarse para informar programas de ejercicio enfocados en personas adultas mayores con enfermedad pulmonar crónica, al ofrecer un insumo sobre la importancia de la combinación efectiva de trabajos específicos y cargas.

Agradecimientos y colaboraciones: al Hospital Nacional de Geriatría y Gerontología, CCSS, por la posibilidad de realizar el estudio.

\section{Referencias}

1. SepÚlveda R. Las enfermedades respiratorias del adulto mayor en Chile: un desafío a corto plazo. Rev Chil Enferm Respir. 2017; 33:303-307.a
2. Sivori M, Almeida M, Benzo R, Boim C, Brassesco M, Callejas O, et al. Nuevo consenso argentino de rehabilitación respiratoria, actualización. Medicina Buenos Aires. 68:325-344.

3. American Association of Cardiovascular \& Pulmonary Rehabilitation (AACVPR). Guidelines for pulmonary rehabilitation programs/American Association of Cardiovascular and Pulmonary Rehabilitation (AACVPR). $4^{\mathrm{a}}$ ed. United States: Human Kinetics, 2011:1-195

4. Agusti A, Decramer M, Celli B, Chen R, Criner G, Frith P, et al. Global strategy for the diagnosis, management, and prevention of chronic obstructive pulmonary disease. 32 páginas. Recuperado el 6 de agosto de 2017. En: https://goldcopd.org/wp-content/uploads/2016/04/wms-spanish-PocketGuide-GOLD-2017.pdf

5. Marco E, Coll-Artés R, Marín M, Coll-Fernández R, Pascual MT, Resa J, et al. Recommendations of the spanish society of cardiorespiratory rehabilitation on pulmonary rehabilitation programmes in patients with chronic obstructive pulmonary disease. Rehabilitacion. 2016; 50: 233-262.

6. Goldstein R, Hill K, Brooks D, Dolmage T. Pulmonary rehabilitation: a review of the recent literature. Chest. 2012; 142:738-749.

7. Güell MR, Díaz S, Rodríguez G, Morante F, San Miguel M, Cejudo, P. et al. Rehabilitación respiratoria. Bronconeumol. 2014; 50: 332-44.

8. Benítez-Pérez R E, Torre-Bouscoulet L, Villca-Alá N, Del-Río-Hidalgo R F, Pérez-Padilla R, Vázquez-García J C, et al. Espirometría: recomendaciones y procedimiento. Neumol Cir Torax. 2016; 75: 173-190.

9. Mottram C D. Ruppel's manual of pulmonary function testing. 11th. United States: ELSEVIER, 2018:1-1237

10. Basso-Vanelli RP, Pires VA, Labadessa, IG, Regueiro EMG, Jamami M, Gomes E. et al. Effects of inspiratory muscle training and calisthenics-and-breathing exercises in COPD with and without respiratory muscle weakness. Respiratory Care. 2016; 61: 50-60.

11. Bolton CE, Bevan EF, Blakey JD, Crowe P, Elkin SL, Garrod R, et al. British thoracic society guideline on pulmonary rehabilitation in adults. Thorax. 2013; 68:1-30.

12. Gosselink R, de Vos J, van den Heuvel SP, Segers J, Decra-mer M, Kwakkel G. Impact of inspiratory muscle training in patients with COPD: What is the evidence? Eur Respir J. 2011;37: 416-425.

13. Mota S. Entrenamiento de los músculos respiratorios en la enfermedad pulmonar obstructiva crónica. En: Guell R, Ramos P. ed. Tratado de rehabilitación respiratoria. Barcelona: Ars Médica, 2010: 221-230.

14. Pinheiro de C, Saldías F. Entrenamiento muscular inspiratorio en el paciente con enfermedad pulmonar obstructiva crónica. Rev Chil Enf Respir. 2011; 27: 116-123.

15. Spruit, M A, Singh SJ, Garvey C, ZuWallack R, Nici L, Rochester, et al. An Official American Thoracic Society/European Respiratory Society Statement: Key concepts and advances in pulmonary rehabilitation. Am J Respir Crit Care Med. 2013; 188:13-264

16. McCarthy B, Casey D, Devane D, Murphy K, Murphy E, \& Lacasse Y. Pulmonary rehabilitation for chronic obstructive pulmonary disease (Review). Cochrane Database of Systematic Reviews. 2015; 2:1-189.

17. Martí J, Vendrell M. Manual SEPAR de procedimientos. Técnicas manuales e instrumentales para el drenaje de secreciones bronquiales en el paciente adulto. 105 páginas. Recuperado el 23 de febrero de 2019. En: https://issuu.com/ separ/docs/manual_separ_27_tecnicas_manuales_?e=3049452/12260872

18. Puhan MA, Gimeno-Santos E, Cates CJ, Troosters T. Pulmonary rehabilitation following exacerbations of chronic obstructive pulmonary disease. Cochrane Database of Systematic Reviews. 2016; 12:1-95.

19. Benzo R, Kelley G, Recchi L, Hofman A, Sciurba F. Complications of lung resection and exercise capacity: a meta-analysis. Respir Med. 2007; 101: 17901797. 